\title{
Temple-Baraitser syndrome
}

INSERM

\section{Source}

INSERM. (1999). Orphanet: an online rare disease and orphan drug data base. TempleBaraitser syndrome. ORPHA:420561

Temple-Baraitser syndrome is a rare developmental anomalies syndrome characterized by severe intellectual disability and distal hypoplasia of digits, particularly of thumbs and halluces, with nail aplasia or hypoplasia. Facial dysmorphism with a pseudo-myopathic appearance has been reported, which may include high anterior hairline or low frontal hairline with central cowlick, flat forehead, ptosis, hypertelorism, downslanting palpebral fissures, epicanthal folds, ears with thick helices, broad depressed nasal bridge with anteverted nares, short columella, long philtrum, high-arched palate, broad mouth with thick vermilion border of the upper or the lower lip and downturned corners. Marked hypotonia, seizures and global developmental delay have been reported, associated with autistic spectrum disorder manifestations in some patients. 